\title{
Ejercicio Físico y Malestar Psicológico en Población Argentina
}

\section{Physical Exercise and Psychological Distress in Argentine Population}

\author{
Francisco González Insua \\ Pontificia Universidad Católica Argentina, Consejo Nacional de Investigaciones Científicas y Técnicas \\ Solange Rodríguez Espínola \\ Pontificia Universidad Católica Argentina \\ Gisela Delfino \\ Pontificia Universidad Católica Argentina, Consejo Nacional de Investigaciones Científicas y Técnicas y \\ Universidad Andres Bello
}

\begin{abstract}
Este estudio tuvo por objetivo analizar el papel del sexo y de la edad como variables moderadoras en la relación entre la frecuencia de realización de ejercicio físico y malestar psicológico en población argentina. La muestra probabilística polietápica consistió en un total de 5388 casos $\left(M_{\text {edad }}=43,15, D E=17,38\right.$, Mínimo $=18$, Máximo $=98 ;$ mujeres $\left.=53,7 \%\right)$. Se efectuaron análisis descriptivos e inferenciales ( $t$ de Student, $\chi^{2}$, ANOVA de un factor, $r$ de Pearson). Se encontró que los hombres realizan más ejercicio físico que las mujeres y que los jóvenes más que los adultos. Se hallaron diferencias en el tipo de ejercicio realizado en función de la edad y el sexo de los participantes. Se verificó que a mayor frecuencia de ejercicio menor es el malestar psicológico. Por último, al efectuar el análisis de moderación para conocer si el efecto positivo del ejercicio físico sobre el malestar varía en función del sexo y/o la edad, se halló que, a mayor edad, mayor es el efecto positivo del mismo. El sexo, sin embargo, no es una variable moderadora en dicha relación.
\end{abstract}

Palabras clave: malestar psicológico, ejercicio físico, edad, sexo, Argentina

\begin{abstract}
The aim of this study was to analyze the role of sex and age as moderating variables in the relationship between the frequency of physical exercise and psychological distress in Argentina. The multi-stage probability sample comprised a total of 5388 cases $\left(M_{\text {age }}=43.15, S D=17.38\right.$, Minimum $=18$, Maximum $=98$; women $\left.=53.7 \%\right)$. Descriptive and inferential analyses (Student's $t$ test, $\chi^{2}$, one-factor ANOVA, Pearson's $r$ ) were performed. It was found that men do more physical exercise than women and that young people exercise more than older people. Also, differences were found in the type of exercise performed depending on the participants' age and sex. It was established that exercising more frequently is associated with lower psychological distress. Finally, a moderation analysis conducted to determine whether the positive effect of physical exercise on distress varies by sex and/or age showed that benefits increase with age. Sex, however, was not found to be a moderating variable in this relationship.
\end{abstract}

Keywords: psychological distress, physical exercise, age, sex, Argentina

Es frecuente que los profesionales de la salud, especialmente los médicos, prescriban la realización de ejercicio físico en pos de mejorar la salud en algunos pacientes, apoyándose en la evidencia científica que existe acerca de ello (Cordero, Masiá \& Galve, 2014; Serra Grima \& Bagur Calafat, 2004). La Organización Mundial de la Salud (OMS) también recomienda indicarlo como herramienta terapéutica en los trastornos mentales comunes, como la ansiedad y la depresión, pero sostiene que la calidad de la evidencia al respecto es baja, por tanto, debe hacerse de forma condicional (Herring, Puetz, O'Connor \& Dishman, 2012; World

Francisco González Insua, Centro de Investigaciones en Psicología y Psicopedagogía, Pontificia Universidad Católica Argentina, Consejo Nacional de Investigaciones Científicas y Técnicas, Buenos Aires, Argentina; Solange Rodríguez Espínola, Observatorio de la Deuda Social Argentina, Pontificia Universidad Católica Argentina, Buenos Aires, Argentina; Gisela Delfino, Centro de Investigaciones en Psicología y Psicopedagogía, Pontificia Universidad Católica Argentina, Consejo Nacional de Investigaciones Científicas y Técnicas, Buenos Aires, Argentina, y Doctorado en Educacion y Sociedad, Facultad de Educación y Ciencias Sociales, Universidad Andrés Bello, Santiago, Chile.

La correspondencia relativa a este artículo debe ser dirigida a Francisco González Insua, Centro de Investigaciones en Psicología y Psicopedagogía, Pontificia Universidad Católica Argentina, Alicia Moreau de Justo 1600 2 Piso, C1107AFD, Ciudad Autónoma de Buenos Aires, Argentina. E-mail: franciscoginsua@uca.edu.ar 
Health Organization [WHO], 2012). Es, justamente, en esta última relación entre ejercicio físico y malestar psicológico en la que se buscó avanzar con la presente investigación.

Cuando se prescribe ejercicio físico, se suele indicar que se practique entre 3-5 días a la semana, que su duración oscile entre un mínimo de 10 a 30 minutos hasta 60 minutos, que sea lo suficientemente significativa como para generar un gasto entre 200 y 300 calorías y que su intensidad esté determinada por las condiciones o estado físico de la persona en cuestión, esperando que se encuentre entre el $50 \%$ y $85 \%$ del consu mo máximo de oxígeno (Jiménez, Martínez, Miró \& Sánchez, 2008).

El uso común y profesional de los términos ejercicio físico y actividad física muestra la necesidad de definirlos y diferenciarlos (Caspersen, Powell \& Christenson, 1985). La actividad física está compuesta por cuatro elementos que la definen: (a) realización de movimientos a través de los músculos del cuerpo, (b) tiene como resultado un gasto de energía, (c) este gasto varía de bajo a alto y (d) guarda una correlación directa con la aptitud física. El ejercicio físico, por otro lado, es un determinado tipo de actividad física que se caracteriza por ser planeada, estructurada y repetitiva y que tiene como objetivo el mantenimiento o mejora de los componentes de la aptitud física. Por su parte, la aptitud física se diferencia de la actividad física y el ejercicio físico. Mientras que actividad y ejercicio hacen referencia a movimientos que los individuos realizan, la aptitud física corresponde a un conjunto de atributos que las personas pueden poseer o desarrollar. Representa atributos relacionados con la salud, como resistencia cardiorespiratoria, resistencia muscular, fuerza muscular, composición corporal y flexibilidad, y otros relacionados con habilidades, como agilidad, balance, coordinación, velocidad y tiempo de reacción. Si bien tanto la actividad como el ejercicio físico guardan una relación directa con la aptitud física, es el ejercicio el que presentaría una asociación mayor con la aptitud física (Caspersen et al., 1985). La presente investigación se centró en el estudio del ejercicio físico y no en el de la actividad física en general.

En Argentina, durante el 2014, el 69\% de los habitantes afirmó no hacer ejercicio físico al menos una vez a la semana, siendo esta inactividad física mayor en las mujeres y en los sujetos entre 35 y 59 años (Rodríguez Espínola, 2015).

Según la OMS, el número de personas con trastornos mentales comunes en todo el mundo está aumentando. Esto se debe a que la población mundial aumenta y más personas viven en la edad en que la depresión y la ansiedad ocurren con mayor frecuencia. Estos trastornos son considerados comunes, porque son altamente prevalentes en la población. Tanto la ansiedad como la depresión tienen un impacto en los sentimientos de las personas afectadas y sus síntomas varían en términos de su gravedad y su duración. Los mismos son condiciones de salud diagnosticables y se diferencian de los sentimientos de tristeza, estrés o temor que cualquier persona puede experimentar ocasionalmente en su vida (WHO, 2017). Sin tener la gravedad de un trastorno psicótico, la ansiedad y la depresión afectan la capacidad para trabajar y relacionarse con los demás, provocando un malestar psicológico significativo, pudiendo, así, influir en la salud física y mental de una persona (Brenlla \& Aranguren, 2010). En Argentina, durante el año 2014 los habitantes que reportaron un mayor malestar psicológico fueron las mujeres y los adultos mayores de 60 años (Rodríguez Espínola, 2015).

Diferentes autores han estudiado la posible relación inversa entre el malestar psicológico y el ejercicio físico (e.g., Ströhle, 2009). Las investigaciones están basadas en diferentes explicaciones. Por un lado, puede que el ejercicio físico reduzca los síntomas depresivos y ansiosos a través de efectos neurobiológicos antidepresivos y ansiolíticos propios de la actividad física. Por otro, la realización de ejercicio físico podría reducir estos síntomas a través de mecanismos psicológicos o sociales (Brière et al., 2018). A estas dos explicaciones podría agregarse la conceptualización de la realización de ejercicio físico como un comportamiento de tipo físicodeportivo u ocio activo para conseguir felicidad (Torres Deik, Moyano-Díaz \& Páez, 2014) o bien como una forma funcional del manejo de la vivencia emocional de regulación fisiológica activa (Páez \& da Costa, 2014).

Los estudios se han realizado en diferentes regiones y con diversas poblaciones. En Estados Unidos se encontró que aquellos sujetos que tenían niveles bajos y medios de ejercicio físico presentaban mayores probabilidades de estar deprimidos que aquellos que tenían niveles altos (Strawbridge, Deleger, Roberts \& Kaplan, 2002). Esta relación también fue hallada en Finlandia: sujetos que se ejercitaban al menos 2 o 3 veces a la semana experimentaban menos depresión, enojo, desconfianza cínica y estrés que aquellos que lo hacían una vez por semana o no lo hacían (Hassmén, Koivula \& Uutela, 2000). En un estudio más reciente sobre una muestra holandesa se halló, de la misma forma que en los trabajos anteriores, que en la población 
general el ejercicio físico regular se asocia a una menor cantidad de síntomas de depresión y ansiedad (De Moor, Boomsma, Stubbe, Willemsen \& de Geus, 2008).

Con la intención de explorar esta relación en un grupo de edad específico, Morgan, Tobar y Snyder (2010) aplicaron un programa de 15 semanas diseñado para acumular 10.000 pasos por día en adultos mayores sedentarios de Estados Unidos. Además de los beneficios físicos, se encontraron mejoras significativas en el bienestar psicológico de los sujetos. También, enfocándose en una muestra de adultos mayores de 60 años en España, se detectó una relación entre la actividad física y el bienestar, moderada por el nivel de independencia de los sujetos, de manera que esta era mayor en aquellos sujetos con niveles de dependencia más elevados (Garatachea et al., 2009. Vagetti et al. (2014) muestran que la actividad física se asocia de forma directa y constante con la calidad de vida general en adultos mayores. De todas formas, la relación de esta con otros dominios de la calidad de vida es más bien moderada e inconsistente y requiere mayor investigación. Entre estos dominios que muestran una asociación moderada con la actividad física se encuentran las relaciones sociales, la salud general, el ambiente y los dolores, entre otros.

Por otro lado, Ströhle (2009) llevó a cabo una revisión con el objetivo de analizar el potencial terapéutico de la actividad y el ejercicio físico sobre la depresión y los trastornos de ansiedad, encontrando que, aunque la relación entre ambas variables ha sido descrita varias veces, no se la ha estudiado de forma prospectiva. Sostiene que la asociación inversa entre la realización de actividad y ejercicio físico y malestar psicológico es evidente, pero que no lo es tanto para los efectos terapéuticos potenciales del ejercicio físico sobre dichos trastornos.

Stephens (1988), a través de un estudio en población general de Canadá y Estados Unidos, encontró que el nivel de actividad física se asocia de forma directa con el bienestar general y humor positivo y de forma inversa con los niveles de ansiedad y depresión. La relevancia de dicho estudio radica en haber tenido en cuenta variables sociodemográficas que pueden tener incidencia en dicha relación. Así, sostiene que la asociación es independiente del nivel socioeconómico y de la salud física de los sujetos, en tanto que es más fuerte en aquellos mayores de 40 años y de sexo femenino. El papel del sexo en esta relación podría estar relacionado, según lo hallado por Torres Deik et al. (2014) en estudiantes universitarios, con que uno de los comportamientos más eficaces que utilizan los hombres para conseguir felicidad es la práctica de ejercicio físico como comportamiento de ocio activo, no siendo así para las mujeres, que se inclinan más por actividades de ocio pasivo y de afiliación social. Distintas revisiones sistemáticas destacan que estos temas no están ampliamente investigados en el área de salud pública y reclaman más trabajos que ofrezcan datos sobre modelos explicativos de la relación entre ejercicio físico y malestar psicológico (Fox, 1999; Netz, Wu, Becker \& Tenenbaum, 2005; Scully, Kremer, Meade, Graham \& Dudgeon, 1998). Por otra parte, Guillén García, Castro Sánchez y Guillén García (1997) sostienen que no todas las formas de ejercicio físico generan los mismos beneficios. Afirman que aquel que genera mayores beneficios es el que afecta grandes masas musculares, con movimientos rítmicos y continuos (correr, caminar, ciclismo, nadar o bailar) y es aeróbico.

En conclusión, la relación inversa entre ejercicio físico y malestar psicológico se ha demostrado en diferentes investigaciones, pero aún no ha sido estudiada en países latinoamericanos y se demandan más estudios que ofrezcan datos sobre modelos explicativos sobre la misma. En este sentido, y considerando que uno de los pocos trabajos que ha tenido en cuenta la presencia de variables sociodemográficas para intentar explicar esta relación es muy antiguo (Stephens, 1988), se planteó como objetivo general analizar la relación entre la frecuencia de ejercicio físico y malestar psicológico en Argentina, analizando el posible papel moderador de la edad y el sexo en la misma. Por otra parte, se han planteado como objetivos específicos de la presente investigación explorar qué tipos de ejercicio físico realizan los sujetos en Argentina, cuáles son los niveles de malestar psicológico de los mismos y comparar las diferencias en función del sexo y la edad de los sujetos. Las mediciones propias de los objetivos específicos se plantearon para ofrecer más datos que permitan comprender la relación entre ambas variables, esperando encontrar diferencias en las mismas según el sexo y la edad de los participantes. En este marco, se plantearon las siguientes hipótesis de trabajo, que buscan confirmar hallazgos previos en población argentina:

H1: la frecuencia de realización de ejercicio físico varía en función del sexo y la edad de los participantes, siendo las mujeres y los sujetos de mayor edad los que menos se ejercitan.

H2: el nivel de malestar psicológico presenta diferencias en función de la edad y el sexo de los sujetos, siendo mayor en las mujeres y en los adultos mayores.

H3: existe una relación inversa entre la frecuencia de realización de ejercicio físico y el malestar psicológico reportados por los participantes. 
H4: el sexo y la edad tienen un papel moderador en la relación entre ejercicio físico y malestar psicológico, siendo la relación más fuerte en los hombres y en los adultos mayores.

\section{Método}

El diseño de la investigación fue transversal, retrospectivo ex-post-facto con un solo grupo y múltiples medidas (Montero \& León, 2007).

\section{Participantes}

La técnica de selección del muestreo en población de 18 años y más fue representativa de conglomerados urbanos de 80.000 habitantes o más de toda Argentina. El procedimiento de muestreo fue polietápico, con una primera etapa de conglomeración y una segunda de estratificación. En el primer criterio de estratificación se definieron los dominios de análisis, de acuerdo a la región y al tamaño de población de los conglomerados a incluir en la muestra. Siguiendo este criterio, se buscó obtener resultados representativos y con márgenes de error y coeficientes de variación al menos a nivel de cada uno de los grandes centros urbanos (> 800 mil habitantes) y del agrupamiento de centros urbanos medianos (entre 240 mil y 800 mil habitantes) y pequeños $(<240$ mil habitantes y $>50$ mil). Un segundo criterio de estratificación apuntó a mejorar la representación socioeconómica de los hogares, obtenido sobre información del Censo Nacional 2010. El mismo consistió en la elaboración de un índice socioeconómico simple elaborado con cinco indicadores: \% de hogares con hacinamiento, \% de hogares sin cloaca, \% de hogares sin agua dentro de la vivienda, \% de hogares con jefe sin educación secundaria completa y \% de hogares con población de 15-24 años que no estudia ni trabaja.

En la primera etapa, el dominio total del estudio se dividió en dos subdominios: (a) áreas metropolitanas y (b) resto urbano del interior. En el primer subdominio, se relevaron siete conglomerados representativos de la realidad urbana del país, cuyos habitantes y hogares constituyen la población de estudio de la Encuesta de la Deuda Social Argentina (EDSA-Bicentenario), que en 2010 era prácticamente la mitad de la población de la Argentina. Los siete aglomerados urbanos son: Gran Buenos Aires, Ciudad Autónoma de Buenos Aires, Conurbano Bonaerense, Gran Rosario, Gran Córdoba, Gran Mendoza y Gran Tucumán. En el segundo subdominio, se establecieron dos grupos, de acuerdo con el volumen poblacional: ciudades o aglomerados de entre 80.000 y 200.000 habitantes y ciudades o aglomerados de 200.000 habitantes o más. En cada grupo se seleccionaron ciudades, mediante un muestreo aleatorio, con probabilidades proporcionales al tamaño de cada ciudad o aglomerado. De este modo, en el primer estrato quedaron seleccionadas Mar del Plata, Gran Salta, Gran Paraná, Gran Resistencia, Gran San Juan y Neuquén-Plottier-Cipolletti; en el segundo, Zárate, La Rioja, Goya, San Rafael, Comodoro Rivadavia y Ushuaia-Río Grande. Las estimaciones que realiza la EDSA-Bicentenario son representativas del total del subdominio, pero no de cada una de las ciudades o aglomerados que lo componen.

En la segunda etapa, se eligieron radios censales (unidades secundarias de muestreo) dentro de cada aglomerado urbano, con una estrategia de estratificación en pos de minimizar los coeficientes de variación de las principales estimaciones a realizar. La variable utilizada como criterio de estratificación fue el promedio de años de educación del jefe del hogar por radio censal. El total de radios censales o puntos de muestreo seleccionados fueron 952, divididos en cinco estratos en el caso de los aglomerados con 200.000 habitantes o más y en tres estratos en los aglomerados con menos de 200.000 habitantes. Con el propósito de optimizar la captación de los casos de mayor y menor nivel socioeducativo, los cinco estratos de mayor población se dividieron en tres grupos centrales (25\% de los casos cada uno) y dos grupos en los extremos (12,5\% por grupo).

La tercera etapa consistió en la selección sistemática de viviendas y hogares (unidades terciarias). Se estableció un tamaño de muestra de 5.655 hogares, con una asignación esperada de seis hogares por punto. El total de hogares se distribuyó entre los aglomerados con un criterio de no proporcionalidad, a efectos de reducir los márgenes de error muestral. El número de radios asignados en cada aglomerado dependió de la manera en que se determinaron los dominios de representatividad estadística y de la necesidad de predicar sobre cada dominio, dependiendo del número de hogares esperado en cada caso. La selección de los radios muestrales dentro de cada conglomerado y estrato fue aleatoria y ponderada por la cantidad de hogares de cada radio.

Finalmente, en la cuarta etapa se apuntó a la segunda población a describir, las personas de 18 años o más, quienes respondieron por sí mismas y por el hogar del que formaban parte. Una vez seleccionados los 
hogares, el entrevistador seleccionaba a los participantes mediante un sistema de cuotas por sexo y grupo etario, de acuerdo con la estructura demográfica determinada por los datos censales.

El error muestral considerado fue de $+/-1,3 \%$, con una estimación de una proporción poblacional del $50 \%$ y un nivel de confianza del 95\% (Salvia et al., 2015). La EDSA-Bicentenario, al ser una muestra multipropósito, estudia muchas variables. Por lo tanto, no existe un único margen de error muestral. Cada estimación cuenta con su propio margen de error, el cual depende de tres aspectos centrales: la varianza o dispersión del indicador a estimar, el intervalo de confianza de las estimaciones y el tamaño de la muestra y de las submuestras (en caso de examinar categorías específicas).

La muestra consistió en un total de 5388 casos. Un 46,3\% $(n=2497)$ fueron varones y el $53,7 \%(n=2891)$ restante, mujeres. La edad de los sujetos se dividió en tres grupos: jóvenes (de 18 a 34 años), adultos de edad media (de 35 a 59 años) y adultos mayores (mayores a 60 años, con un máximo de 98 años). El primer grupo compone el $38,7 \%$ de la muestra $(n=2086)$, con una media de 26,04 años $(D E=4,83)$; el segundo, el 40,8\% $(n=2200)$, con una media de 46,1 años $(D E=7,21)$, y el tercero, el 20,5\% ( $n=1102)$ del total, con una media de 69,68 años $(D E=7,2)$. La media de edad del total de la población fue de 43,15 $(D E=17,38$; Mínimo = 18; Máximo $=98$ ). Si bien la categorización en estos tres grupos etarios puede presentar limitaciones (se podrían hacer más grupos o grupos con cantidades iguales de participantes), se optó por usar esta división a fin de poder comparar los resultados con estudios previos efectuados sobre muestras representativas de Argentina (Rodríguez Espínola, 2015).

\section{Instrumentos}

Encuesta de la Deuda Social Argentina (EDSA-BICENTENARIO, 2010-2016; Salvia et al., 2015). Es una encuesta multipropósito integrada por distintas preguntas y secciones. Aquí solo se reportan las relativas a los objetivos planteados.

Para conocer la frecuencia de realización de ejercicio físico se consultó: “¿Con qué frecuencia usted realiza ejercicio físico (gimnasia, deportes, caminatas, etc.)?”, pudiendo los participantes indicar alguna de las siguientes opciones: $1=$ nunca o de manera casual, $2=$ algunas veces al mes, $3=$ al menos una vez a la semana, $4=$ dos o tres veces por semana, $5=$ más de tres veces por semana.

En relación al tipo de ejercicio físico que suelen realizar, se presentó una frase que debían completar: "Cuando usted realiza un ejercicio físico, realiza un...", categorizándose las respuestas en cuatro alternativas: Deporte individual, Deporte grupal, Ejercicio físico guiado por profesor y Ejercicio físico guiado sin profesor.

Escala de Malestar Psicológico de Kessler (Kessler Psychological Distress Scale, adaptación argentina realizada por Brenlla \& Aranguren, 2010). Se utilizó para evaluar el nivel de malestar psicológico. La KPDS-10 (por sus siglas en inglés) es una versión abreviada que cuenta con 10 ítems que miden un conjunto de síntomas relacionados con depresión (e.g., ¿Usted se sintió deprimido en el último mes?) y ansiedad (e.g., ¿Usted se ha sentido tan nervioso/a que nada podía calmarlo/a en el último mes?), como desesperanza, tristeza, nerviosismo y cansancio. El entrevistado debe responder a cada ítem teniendo en cuenta cómo se sintió en el último mes. El continuo de respuestas es tipo Likert de cinco puntos: $5=$ todo el tiempo, $4=$ la mayor parte del tiempo, $3=$ a veces, $2=$ pocas veces y $1=$ nunca. Según el puntaje total obtenido, los resultados de esta escala se agrupan en cuatro categorías: alto malestar (valores entre 4,1 y 5), moderado malestar (valores entre 3,1 y 4), bajo malestar (valores entre 2,1 y 3 ) y sin malestar (valores entre 1 y 2). El análisis de las características psicométricas realizado en la adaptación a población argentina brinda evidencias acerca de la confiabilidad y validez de constructo, muchas de las cuales son consistentes con los resultados de estudios previos realizados en muestras de población general (Baggaley et al., 2007; Fassaert et al., 2009; Kessler et al., 2003). En relación a la consistencia interna de la escala, Brenlla y Aranguren (2010) reportaron valores alfa de Cronbach $(0,88)$ coincidentes con los informados por otras investigaciones en diferentes países. Obtuvieron un solo factor que explica el 45,5\% de la varianza total; todos los ítems cargan en el primer factor, concluyendo en la unidimensionalidad del constructo medido, esto es, el riesgo de malestar psicológico inespecífico. En el presente estudio se obtuvo un alfa de Cronbach = 0,91.

\section{Procedimiento}

La recolección de los datos se realizó en el último trimestre del 2015. La participación fue voluntaria, pudiendo el encuestado omitir, no informar o culminar la encuesta si lo creía necesario. Antes de comenzar 
la encuesta, los participantes debían firmar un consentimiento informado, que cumple con los lineamientos para el comportamiento ético para las Ciencias Sociales y Humanas establecidos por el Consejo Nacional de Investigaciones Científicas y Técnicas de Argentina (Resolución № 2857/06), en el que se informaba, además, acerca de los propósitos de la investigación.

\section{Análisis de Datos}

No se efectuó ningún tratamiento especial a los datos perdidos ni se eliminaron participantes. No se registraron datos perdidos en relación a la edad y al sexo. Para el malestar psicológico y el ejercicio físico, los datos perdidos no alcanzaron un 1,5\%, por tanto, no afectaron los resultados. Se efectuaron análisis descriptivos para la frecuencia de realización de ejercicio físico, el tipo de ejercicio físico realizado y el nivel de malestar psicológico, reportándose porcentajes y medias según el caso. Aunque tanto en frecuencia de ejercicio físico como en malestar psicológico las categorías eran ordinales, para realizar los análisis se calcularon las medias. Para el cálculo de las medias de frecuencia de ejercicio físico se consideró $1=$ nunca o de manera casual y $5=$ más de tres veces por semana, y para el malestar psicológico, desde $1=\sin$ malestar a $4=$ alto malestar. Para comparar las mismas variables según sexo se utilizaron los estadísticos $t$ de Student y $\chi^{2}$. En lo que respecta a los grupos de edad, se efectuaron análisis de varianza (ANOVA) de un factor y $\chi^{2}$. Con el objetivo de analizar la relación entre la frecuencia de realización de ejercicio físico y malestar psicológico se usó la prueba $r$ de Pearson. Se utilizaron estadísticos paramétricos ya que, según la teoría central del límite, se asume una distribución normal cuando el tamaño muestral es grande (Szretter Noste, 2017). En el caso de los ANOVA, la prueba post-hoc utilizada fue T3 de Dunnett. Para estos cálculos se utilizó el paquete estadístico SPSS v. 20.0. Se calculó el tamaño del efecto para cada uno de los contrastes efectuados. Para la prueba $t$ de Student se utilizó el estadístico $d$ de Cohen, para el ANOVA se aplicó el estadístico $f$ de Cohen y para la prueba $\chi^{2}$ se empleó el estadístico $w$. Para efectuar estos cálculos se utilizó el paquete estadístico G*Power v. 3.1.9.2 (Cárdenas Castro \& Arancibia Martini, 2014; Faul, Erdfelder, Buchner \& Lang, 2009). Finalmente, con la intención de examinar el posible efecto moderador de las variables sexo y edad en la relación entre la frecuencia de realización de ejercicio físico y malestar psicológico se aplicó la macro PROCESS (modelo 2) para SPSS desarrollada por Hayes (2018). Esta macro permite efectuar un análisis de trayectoria (path) basado en la regresión. Específicamente, el modelo utilizado analiza la influencia del sexo y la edad (moderadores) en el tamaño del efecto de la frecuencia de ejercicio físico sobre el malestar psicológico.

\section{Resultados}

\section{Frecuencia de Realización de Ejercicio Físico}

Como se exhibe en la Tabla 1, un 54\% nunca efectúa ejercicio físico o lo hace de forma casual, siendo solo un $8 \%$ los que declaran realizarlo más de tres veces por semana. Se encontraron diferencias con un tamaño del efecto pequeño en función del sexo, $t(5178,9)=6,76, p<0,001,95 \%$ IC $[0,19,0,34], d=0,27$, y del grupo etario, $F(2,5329)=77,43, p<0,001 ; 95 \%$ IC $[2,10,2,17], f=0,17$. Como lo indica la hipótesis 1 , los hombres tienden a realizar ejercicio físico con mayor frecuencia que las mujeres, mientras que los más jóvenes son más activos que los adultos de edad media $(p<0,001)$ o los adultos mayores $(p<0,001)$.

Tabla 1

Frecuencia de Realización de Ejercicio Físico, Según Sexo y Grupo Etario (en Porcentaje)

\begin{tabular}{|c|c|c|c|c|c|c|}
\hline Frecuencia & Total & Hombres & Mujeres & $\begin{array}{c}\text { Jóvenes } \\
\text { (18 a } 34 \\
\text { años) }\end{array}$ & $\begin{array}{l}\text { Adultos } \\
\text { (35 a } 59 \\
\text { años) }\end{array}$ & $\begin{array}{c}\text { Adultos } \\
\text { mayores (60 } \\
\text { años o más) }\end{array}$ \\
\hline Nunca o casual & 53,7 & 47,3 & 59,2 & 41,0 & 58,6 & 67,8 \\
\hline Algunas veces al mes & 11,1 & 12,6 & 9,8 & 14,3 & 10,8 & 5,6 \\
\hline Una vez por semana & 11,5 & 14,7 & 8,8 & 14,8 & 10,6 & 7,0 \\
\hline Dos o tres veces por semana & 15,4 & 16,2 & 14,8 & 20,8 & 12,0 & 12,1 \\
\hline Más de tres veces por semana & 8,3 & 9,3 & 7,4 & 9,1 & 7,9 & 7,4 \\
\hline
\end{tabular}




\begin{tabular}{lcccccc}
$n$ & 5388 & 2497 & 2891 & 2086 & 2200 & 1102 \\
Media $(D E)$ & $2,14(1,41)$ & $2,28(1,42)$ & $2,01(1,39)$ & $2,42(1,41)$ & $2,00(1,37)$ & $1,86(1,37)$ \\
\hline
\end{tabular}

\section{Tipo de Ejercicio Físico Realizado}

Respecto del tipo de ejercicio físico, tres de cada cinco encuestados optan por un ejercicio físico sin un profesor que los guíe (ver Tabla 2). La frecuencia del deporte en equipo es mayor en los hombres que en las mujeres, $\chi^{2}(3, N=3680)=466,86, p<0,001$, ya que ellas participan más en ejercicios físicos tanto guiados o no guiados por profesor, como puede ser el gimnasio, clases de gimnasia o bicicleta. El tamaño del efecto de esta diferencia es grande, $w=0,54$. En lo que respecta a los grupos de edad, se observa que el deporte individual se mantiene equitativamente en los distintos grupos, mientras que el deporte en equipo es elegido más frecuentemente en la medida que se es más joven, $\chi^{2}(6, N=3680)=330,13, p<0,001$. La ejercitación sin la guía de un profesor aumenta a medida que se incrementa la edad, ya que crece desde un $45 \%$ en los jóvenes hasta alcanzar un $78 \%$ en los adultos mayores. El tamaño del efecto de esta diferencia es mediano, $w=0,31$.

Tabla 2

Tipo de Ejercicio Físico Realizado, Según Sexo y Grupo Etario (en Porcentaje)

\begin{tabular}{|c|c|c|c|c|c|c|}
\hline Tipo de ejercicio físico & Total & Hombres & Mujeres & $\begin{array}{c}18 \text { a } 34 \\
\text { años }\end{array}$ & $\begin{array}{c}35 \text { a } 59 \\
\text { años }\end{array}$ & $\begin{array}{c}60 \text { años o } \\
\text { más }\end{array}$ \\
\hline Deporte individual & 6,8 & 6,7 & 7,0 & 7,2 & 6,3 & 7,1 \\
\hline Deporte en equipo & 17,3 & 30,7 & 4,1 & 27,5 & 12,2 & 2,8 \\
\hline Ejercicio físico guiado por profesor & 16,3 & 11,9 & 20,6 & 20,1 & 14,1 & 11,7 \\
\hline Ejercicio físico no guiado por profesor & 59,6 & 50,7 & 68,3 & 45,3 & 67,4 & 78,4 \\
\hline$n$ & 5388 & 2497 & 2891 & 2086 & 2200 & 1102 \\
\hline
\end{tabular}

Por otro lado, al analizar el tipo de ejercicio físico realizado en función del grupo etario al interior de cada sexo, se encuentra que existen diferencias tanto en hombres, $\chi^{2}(6, N=2497)=297,27, p<0,001$, como en mujeres, $\chi^{2}(6, N=2891)=57,37, p<0,001$. El deporte en equipo es elegido mayormente por los hombres jóvenes y en segunda instancia por los hombres adultos de edad media. En todos los grupos de edad, el ejercicio físico guiado por profesor es efectuado en su mayoría por mujeres. Conforme aumenta la edad también lo hace la práctica del ejercicio físico sin la guía de un profesor, independientemente del sexo (ver Tabla 3). Al considerar el tamaño del efecto, se puede afirmar que las diferencias en el grupo de los hombres en función del grupo etario son mayores, $w=0,55$, que en las mujeres, $w=0,22$.

Tabla 3

Tipo de Ejercicio Físico en Función del Sexo y Grupo Etario (en Porcentaje)

Hombres Mujeres

Tipo de ejercicio físico

\begin{tabular}{lrrrrrr}
\cline { 2 - 6 } & $\begin{array}{c}18 \text { a } 34 \\
\text { años }\end{array}$ & $\begin{array}{c}35 \text { a } 59 \\
\text { años }\end{array}$ & $\begin{array}{c}60 \text { años y } \\
\text { más }\end{array}$ & $\begin{array}{c}18 \text { a } 34 \\
\text { años }\end{array}$ & $\begin{array}{c}35 \text { a } 59 \\
\text { años }\end{array}$ & $\begin{array}{c}60 \text { años o } \\
\text { más }\end{array}$ \\
\hline Deporte individual & 7,2 & 6,5 & 5,4 & 7,2 & 6,1 & 8,6 \\
Deporte en equipo & 45,4 & 23,9 & 3,7 & 7,3 & 1,9 & 2,1 \\
Ejercicio físico guiado por profesor & 16,0 & 7,9 & 9,2 & 24,7 & 19,5 & 13,9 \\
Ejercicio físico no guiado por profesor & 31,4 & 61,7 & 81,6 & 60,9 & 72,5 & 75,5 \\
$N$ & 1016 & 992 & 489 & 1070 & 1208 & 613 \\
\hline
\end{tabular}




\section{Malestar Psicológico}

Según los datos reportados en la Tabla 4, un 9,4\% de la población total expresa un alto o moderado malestar psicológico, siendo únicamente el 0,8\% aquellos que padecen alto malestar. En línea con lo esperado, se hallaron diferencias según el sexo, $t(5294,78)=-5,06, p<0,001,95 \%$ IC $[-0,13,-0,05]$, y la edad, $F(2,5319)=13,39, p<0,001,95 \%$ IC $[1,40,1,44], f=0,07$. En lo que respecta al sexo, el tamaño del efecto es pequeño, $d=0,13$ : las mujeres padecen mayor malestar que los hombres, superándolos en los grupos de moderado y bajo malestar. En cuanto a la edad, también el tamaño del efecto es pequeño: los jóvenes presentan un mayor porcentaje de sujetos sin malestar psicológico que los adultos, tanto de mediana edad $(p<0,001)$ como mayores $(p<0,001)$.

Tabla 4

Niveles de Malestar Psicológico, Según Sexo y Grupo Etario (en Porcentaje)

\begin{tabular}{lcccccc}
\hline Nivel & Total & Hombres & Mujeres & $\begin{array}{l}\text { 18 a } 34 \\
\text { años }\end{array}$ & $\begin{array}{l}\text { 35 a } 59 \\
\text { años }\end{array}$ & $\begin{array}{l}\text { 60 años o } \\
\text { más }\end{array}$ \\
\hline Alto & 0,8 & 0,7 & 1,0 & 0,8 & 0,8 & 1,0 \\
Moderado & 8,6 & 7,5 & 9,6 & 7,1 & 9,3 & 10,2 \\
Bajo & 22,5 & 20,1 & 24,6 & 19,7 & 24,6 & 23,6 \\
Sin malestar psicológico & 68,0 & 71,7 & 64,9 & 72,4 & 65,3 & 65,2 \\
$n$ & 5388 & 2497 & 2891 & 2086 & 2200 & 1102 \\
Media $(D E)$ & $1,42(0,68)$ & $1,37(0,65)$ & $1,47(0,70)$ & $1,36(0,65)$ & $1,46(0,70)$ & $1,47(0,72)$ \\
\hline
\end{tabular}

\section{Ejercicio Físico y Malestar Psicológico}

Como era esperado, se halló que a mayor frecuencia de realización de ejercicio físico menor es el nivel de malestar psicológico reportado, $r=-0,080, p<0,001, n=5267$. De todas formas, este coeficiente de correlación es muy pequeño.

Teniendo en cuenta que se verificaron diferencias en función del sexo y de la edad en cuanto a la frecuencia de realización de ejercicio físico y el malestar psicológico reportado, se efectuó un análisis de moderación (en base a la regresión) para conocer si el efecto positivo del ejercicio físico sobre el malestar varía en función del sexo y/o la edad de los participantes.

En la Tabla 5 se presentan los resultados obtenidos. Como puede observarse, la edad tiene un efecto moderador, aunque muy pequeño, en la relación entre la frecuencia de realización de ejercicio físico y malestar psicológico. Este efecto no se verifica para la variable sexo. En este caso, la hipótesis planteada no se confirma, ya que solo la variable edad tiene un papel moderador y no ambas, como era esperado.

Tabla 5

Análisis del Efecto Moderador del Sexo y la Edad en la Relación entre Frecuencia de Realización de Ejercicio Físico y Malestar Psicológico

\begin{tabular}{lccrr}
\hline \multicolumn{1}{c}{ Variable predictora } & $\mathrm{B}$ & $E E$ & \multicolumn{1}{c}{$t$} & \multicolumn{1}{c}{$p$} \\
\hline Constante & 1,32 & 0,05 & 28,00 & $<0,001$ \\
Sexo & 0,05 & 0,03 & 1,49 & 0,135 \\
Frecuencia de ejercicio físico & 0,01 & 0,02 & 0,32 & 0,750 \\
Interacción Frecuencia de ejercicio físico x Sexo & 0,01 & 0,01 & 1,08 & 0,281 \\
Edad & 0,01 & 0,01 & 3,94 & $<0,001$ \\
Interacción Frecuencia de ejercicio físico x Edad & $-0,01$ & 0,01 & $-2,33$ & 0,020 \\
\hline
\end{tabular}

Nota. La variable sexo se recodificó como variable dummy, asignando el valor 0,5 a mujeres y -0,5 a hombres, siguiendo las recomendaciones de Hayes (2018). B: coeficiente de regresión no estandarizado; $E E$ : error estándar. $R^{2}=0,014$, $F(5,5261)=14,46, p<0,001$. 
En la Figura 1 se sintetizan los resultados obtenidos para cada uno de los seis grupos que se conforman al considerar el sexo y la edad. Al comparar la fuerza de la relación inversa entre la frecuencia de realización de ejercicio físico y el malestar psicológico reportado, se observa que en los adultos de mayor edad esta es más alta $\left(M_{\text {edad }}=60,53\right)$, Mujeres: $B=-0,041, p<0,001$; Hombres: $B=-0,056, p<0,001$. Esta relación es menor en los adultos de mediana edad $\left(M_{\text {edad }}=43,15\right)$, Mujeres: $B=-0,026, p=0,005$; Hombres: $B=-0,040$, $p<0,001$, y aún menor en los más jóvenes $\left(M_{\text {edad }}=25,76\right)$, Mujeres: $B=-0,010, p=0,370$; Hombres: $B=-0,025, p=0,032)$. De esta forma, el efecto de la realización de ejercicio físico sobre la disminución del malestar psicológico es mayor, si bien siempre es pequeño, conforme aumenta la edad, independientemente del sexo de los participantes.

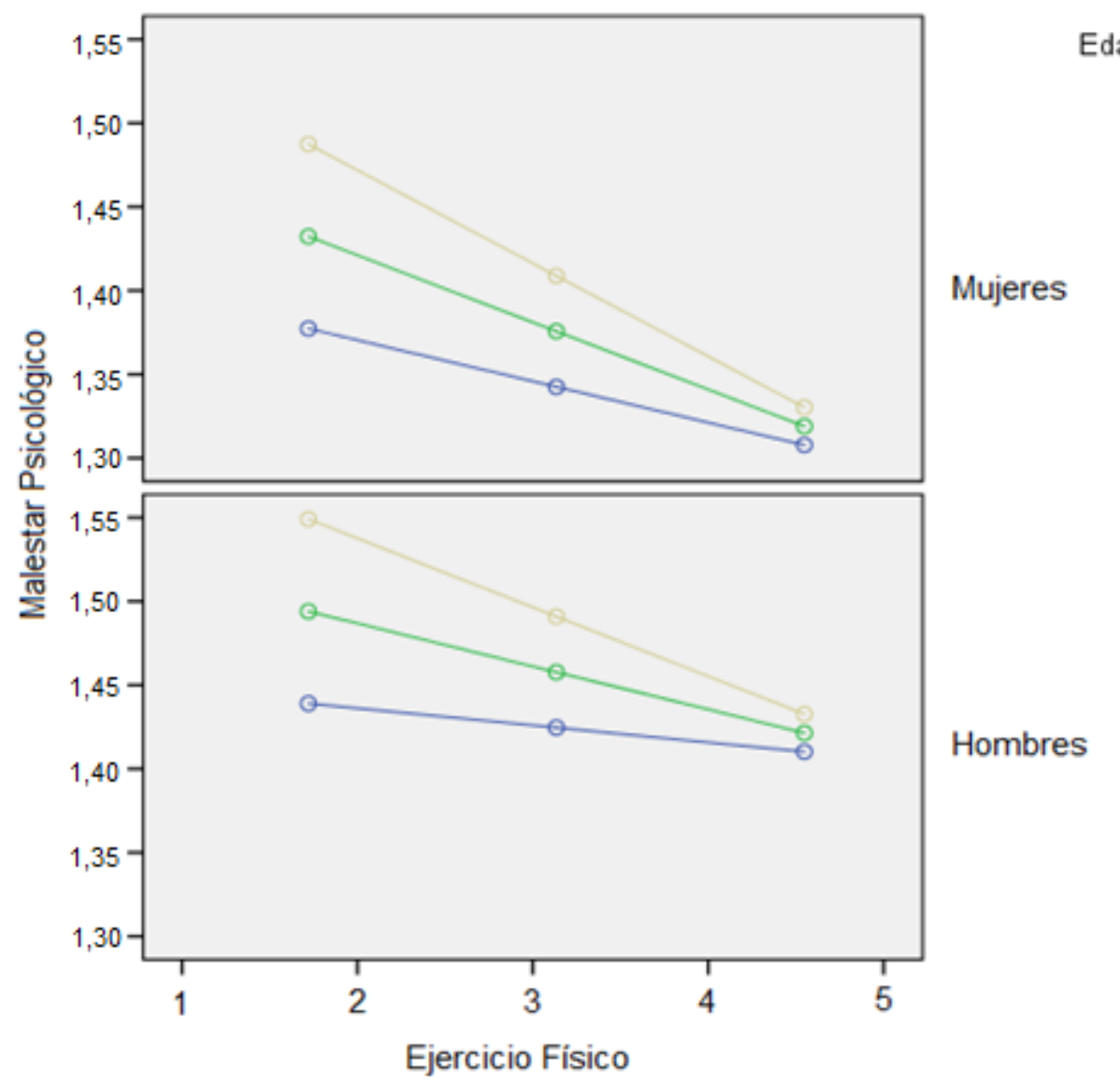

Figura 1. Relación entre frecuencia de realización de ejercicio físico y malestar psicológico, según edad y sexo. Las medias de edad utilizadas no son las de los grupos etarios planteados a lo largo del trabajo, sino que se corresponden con - $1 D E$, media de la muestra total y $+1 D E$.

\section{Discusión}

Si bien los datos confirman las hipótesis planteadas, los tamaños del efecto son pequeños. Se verificaron pequeñas variaciones en la frecuencia de realización de ejercicio físico a favor de los hombres y de los más jóvenes (hipótesis 1) y en el nivel de malestar psicológico, siendo las mujeres y los adultos mayores a 60 años los más afectados (hipótesis 2). Más interesante, se constató una pequeña relación entre la mayor frecuencia de realización de ejercicio físico y la disminución del malestar psicológico (hipótesis 3) y se halló que esta presenta un ligero incremento conforme aumenta la edad de los participantes (hipótesis 4). La hipótesis 4 también planteaba una variación en función del sexo, la que no pudo ser confirmada. Estos resultados 
confirman lo señalado por estudios previos efectuados en otros contextos culturales (De Moor et al., 2008; Garatachea et al., 2009; Hassmén et al., 2000; Morgan et al., 2010; Strawbridge et al., 2002), pero el bajo tamaño del efecto señala la importancia de ser cautelosos a la hora de efectuar afirmaciones sobre la relación entre frecuencia de realización de ejercicio físico y malestar psicológico o la importancia de las diferencias en función del sexo y la edad, ya que las mismas podrían deberse al gran tamaño de la muestra. En cualquier caso, es importante considerar la relevancia clínica de los resultados: la mayor frecuencia de realización de ejercicio podría tener un efecto paliativo, aunque bajo, en el malestar psicológico de la población — tanto en hombres como en mujeres-, especialmente en aquellos individuos de mayor edad.

A diferencia de los hallazgos de Stephens (1988) que planteaban una relación más fuerte entre la frecuencia de realización de ejercicio físico y la disminución del malestar psicológico en las mujeres, en comparación con los hombres, en este estudio no se encontró que el sexo genere diferencias en la fuerza de la relación. Sin embargo, los resultados coinciden en el papel moderador de la edad en esta relación, ya que, en ambos estudios - la muestra utilizada en el estudio de Stephens (1988) también era muy grande-, a medida que aumenta la edad la relación es mayor. Torres Deik et al. (2014) hallaron que los hombres encuentran en el ejercicio físico un comportamiento de ocio activo que les permite conseguir felicidad, mientras que las mujeres prefieren actividades de ocio pasivo o afiliación social. En este sentido, resulta llamativo que el sexo no funcione como una variable moderadora en la relación entre ejercicio físico y malestar psicológico, ya que se esperaba que los hombres consiguieran mayores beneficios de este que las mujeres.

Si bien no se formuló ninguna hipótesis relativa a las posibles diferencias en el tipo de ejercicio físico preferido en función del sexo o la edad, los resultados obtenidos plantean la importancia de considerarlas. Tres de cada 10 hombres prefieren el deporte en equipo, mientras que apenas una de cada 25 mujeres opta por este tipo de ejercicio. Al considerar las diferencias en función de la edad, con una fuerza media, se encontró que el deporte en equipo es popular entre los jóvenes, mientras que entre los de mayor edad prevalece la ejercitación sin la guía de un profesor. Se sabe que los adultos mayores tienden a percibir menor apoyo social que otros grupos etarios (Aceiro, González Insua, Grasso \& Delfino, 2017) y la disminución en la preferencia por la realización de deporte en equipo podría estar asociada con esta percepción. En otras palabras, si el adulto percibe que su red social es escasa o pequeña, podría encontrar problemas a la hora de participar en una actividad que requiere de varios sujetos para ser llevada a cabo. Este estudio muestra que el deporte en equipo es ampliamente elegido por los hombres jóvenes y también que el ejercicio físico sin guía de profesor es elegido mayormente por mujeres, independientemente de su edad. Este hallazgo resulta interesante y considerarlo podría contribuir a generar una mayor adherencia al tratamiento en aquellos pacientes a los que se les prescribe ejercicio físico para conseguir beneficios en su salud, ya que se podrá conocer qué tipo de ejercicio físico es elegido por el grupo al cual pertenecen.

La OMS (WHO, 2017) demuestra un aumento del número de personas con trastornos mentales comunes en todo el mundo y sostiene que esto se debe a que la población mundial ha aumentado, al tiempo que más personas viven en la edad en que la depresión y la ansiedad ocurren con mayor frecuencia. Los resultados obtenidos van en esta línea: la mayor concentración de individuos con síntomas de ansiedad y depresión se encuentra en los adultos mayores a 60 años, específicamente en las mujeres de dicho grupo. Esto replica los resultados hallados en 2014 en Argentina, ya que también los participantes que reportaron un mayor malestar psicológico fueron las mujeres y aquellos mayores de 60 años (Rodríguez Espínola, 2015). En consecuencia, incentivar la realización de ejercicio físico en esta población, tomando los recaudos necesarios, podría conllevar una pequeña disminución en su malestar psicológico. Sin duda, la vulnerabilidad de este sector de la sociedad pone de relieve la importancia del desarrollo de políticas públicas dirigidas hacia el mismo.

Una de las limitaciones de esta investigación está relacionada con que el cuestionario no especificaba otras formas de ejercicio físico más que las expresadas en el ítem que indagaba acerca de la frecuencia del mismo, por tanto, es posible que los participantes estuviesen pensando en otros tipos al momento de contestar. Además, las opciones de respuesta al ítem no ofrecían categorías excluyentes, por lo que un sujeto podría incluirse en dos grupos al mismo tiempo. Por otro lado, al ser una muestra grande evaluada en un contexto de encuesta y con un cuestionario multipropósito, la frecuencia y el tipo de ejercicio físico fueron explorados con un ítem único de autorreporte, pudiendo verse influido por la deseabilidad social de los participantes. Por ello, podría ser interesante utilizar medidas objetivas, con los dispositivos tecnológicos específicos, para medir exactamente cuál es el nivel de ejercicio físico que realizan los sujetos y explorar la relación con el malestar psicológico. 
A partir de los resultados del presente estudio surgen diferentes interrogantes que podrían dar lugar a investigaciones futuras: ¿la realización de ejercicio físico puede generar la disminución del malestar psicológico? Si las mujeres eligieran la práctica de ejercicio físico como estrategia de afrontamiento al malestar psicológico, ¿encontrarían los mismos resultados que con otras actividades de ocio pasivo? El hábito de ejercitarse físicamente ise puede desarrollar en una edad avanzada o debe cultivarse desde la juventud? Aquí se vuelve a poner en evidencia la necesidad de estudiar la relación entre ambas variables de forma prospectiva, como bien lo indicó Ströhle (2009). Por último, ¿qué otras variables, además de la edad, moderan la relación entre el ejercicio físico y el malestar psicológico?

En síntesis, la presente investigación aporta información acerca de la relación entre el ejercicio físico y el malestar psicológico en el contexto argentino. Se ha encontrado una débil relación inversa entre ambas variables que parecería ser mayor conforme aumenta la edad, independientemente del sexo de los participantes. Si bien el sexo y la edad generarían pocas diferencias en la frecuencia de realización de ejercicio físico y en los niveles de malestar, sí arrojan diferencias al considerar el tipo de ejercicio físico preferido. Los hallazgos replican la literatura previa al respecto, quedando en evidencia la necesidad de continuar estudiando esta relación, intentando explicar la forma en la que se asocian ambas variables.

\section{Referencias}

Aceiro, M. A., González Insua, F., Grasso, L. \& Delfino, G. I. (2017). Apoyo social percibido a lo largo del ciclo vital: su relación con indicadores de ansiedad y depresión en la vejez. Memorias de las Jornadas de Investigación, 24(2), 128. Extraído de https://www.aacademica.org/000-067/384

Baggaley, R. F., Ganaba, R., Filippi, V., Kere, M., Marshall, T., Sombié, I. ... Patel, V. (2007). Detecting depression after pregnancy: The validity of the K10 and K6 in Burkina Faso. Tropical Medicine \& International Health, 12, $1225-1229$. https://doi.org/10.1111/j.1365-3156.2007.01906.x

Brenlla, M. E. \& Aranguren, M. (2010). Adaptación argentina de la Escala de Malestar Psicológico de Kessler (K10). Revista de Psicología de la Pontificia Universidad Católica del Perú, 28, 308-340. Extraído de https://dialnet.unirioja.es/servlet/articulo?codigo=4637549

Brière, F. N., Yale-Soulière, G., Gonzalez-Sicilia, D., Harbec, M. -J., Morizot, J., Janosz, M. \& Pagani, L. S. (2018). Prospective associations between sport participation and psychological adjustment in adolescents. Journal of Epidemiology \& Community Health, 72, 575-581. https://doi.org/10.1136/jech-2017-209656

Cárdenas Castro, J. M. \& Arancibia Martini, H. (2014). Potencia estadística y cálculo del tamaño del efecto en G*Power: complementos a las pruebas de significación estadística y su aplicación en psicología. Salud \& Sociedad, 5, 210-224. https://doi.org/10.22199/S07187475.2014.0002.00006

Caspersen, C. J., Powell, K. E. \& Christenson, G. M. (1985). Physical activity, exercise, and physical fitness: Definitions and distinctions for health-related research. Public Health Reports, 100, 126-131. Extraído de https://www.ncbi.nlm.nih.gov/pmc/articles/PMC1424733/pdf/pubhealthrep00100-0016.pdf

Cordero, A., Masiá, M. D. \& Galve, E. (2014). Ejercicio físico y salud. Revista Española de Cardiología, 67, 748-753. https://doi.org/10.1016/j.recesp.2014.04.007

De Moor, M. H. M., Boomsma, D. I., Stubbe, J. H., Willemsen, G. \& de Geus, E. J. C. (2008). Testing causality in the association between regular exercise and symptoms of anxiety and depression. Archives of General Psychiatry, 65, 897-905. https://doi.org/10.1001/archpsyc.65.8.897

Fassaert, T., De Wit, M. A. S., Tuinebreijer, W. C., Wouters, H., Verhoeff, A. P., Beekman, A. T. F. \& Dekker, J. (2009). Psychometric properties of an interviewer-administered version of the Kessler Psychological Distress Scale (K10) among Dutch, Moroccan and Turkish respondents. International Journal of Methods in Psychiatric Research, 18, 159-168. https://doi.org/10.1002/mpr.288

Faul, F., Erdfelder, E., Buchner, A. \& Lang, A. -G. (2009). Statistical power analyses using G*Power 3.1: Tests for correlation and regression analyses. Behavior Research Methods, 41, 1149-1160. https://doi.org/10.3758/BRM.41.4.1149

Fox, K. R. (1999). The influence of physical activity on mental well-being. Public Health Nutrition, 2, 411-418. https://doi.org/10.1017/S1368980099000567

Garatachea, N., Molinero, O., Martínez-García, R., Jiménez-Jiménez, R., González-Gallego, J. \& Márquez, S. (2009). Feelings of well being in elderly people: Relationship to physical activity and physical function. Archives of Gerontology and Geriatrics, 48, 306-312. https://doi.org/10.1016/j.archger.2008.02.010

Guillén García, F., Castro Sánchez, J. J. \& Guillén García, M. A. (1997). Calidad de vida, salud y ejercicio físico: una aproximación al tema desde una perspectiva psicosocial. Revista de Psicología del Deporte, 6(2), 91-110. Extraído de https://ddd.uab.cat/pub/revpsidep/19885636v6n2/19885636v6n2p91.pdf

Hassmén, P., Koivula, N. \& Uutela, A. (2000). Physical exercise and psychological well-being: A population study in Finland. Preventive Medicine, 30, 17-25. https://doi.org/10.1006/pmed.1999.0597

Hayes, A. F. (2018). Introduction to mediation, moderation and conditional process analysis. A regression-based approach (2a ed.). New York, NY: Guilford Press.

Herring, M. P., Puetz, T. W., O'Connor, P. J. \& Dishman, R. K. (2012). Effect of exercise training on depressive symptoms among patients with a chronic illness: A systematic review and meta-analysis of randomized controlled trials. Archives of Internal Medicine, 172, 101-111. https://doi.org/10.1001/archinternmed.2011.696

Jiménez, M. G., Martínez, P., Miró, E. \& Sánchez, A. I. (2008). Bienestar psicológico y hábitos saludables: ¿están asociados a la práctica de ejercicio físico? International Journal of Clinical and Health Psychology, 8, 185-202. Extraído de http://digibug.ugr.es/bitstream/handle/10481/32914/Jimenez_PsicologiaEjercicio.pdf?sequence=1\&isAllowed=y

Kessler, R. C., Barker, P. R., Colpe, L. J., Epstein, J. F., Gfroerer, J. C., Hiripi, E. ... Zaslavsky, A. M. (2003). Screening for serious mental illness in the general population. Archives of General Psychiatry, 60, 184-189. https://doi.org/10.1001/archpsyc.60.2.184 
Montero, I. \& León, O. G. (2007). A guide for naming research studies in psychology. International Journal of Clinical and Health Psychology, 7, 847-862. Extraído de http://www.aepc.es/ijchp/GNEIP07_es.pdf

Morgan, A. L., Tobar, D. A. \& Snyder, L. (2010). Walking toward a new me: The impact of prescribed walking 10,000 steps/day on physical and psychological well-being. Journal of Physical Activity \& Health, 7, 299-307. https://doi.org/10.1123/jpah.7.3.299

Netz, Y., Wu, M. -J., Becker, B. J. \& Tenenbaum, G. (2005). Physical activity and psychological well-being in advanced age: A metaanalysis of intervention studies. Psychology and Aging, 20, 272-284. https://doi.org/10.1037/0882-7974.20.2.272

Páez, D. \& da Costa, S. (2014). Regulación afectiva (de emociones y estado de ánimo) en el lugar de trabajo. Revista Psicologia: Organizações \& Trabalho, 14, 190-203. Extraído de http://pepsic.bvsalud.org/scielo.php?script=sci_arttext\&pid=S1984-66572014000200006

Rodríguez Espínola, S. (2015). Condición y prevención de la salud, recursos psicológicos y redes de contención social. En A. Salvia (Coord.), Progresos sociales, pobrezas estructurales y desigualdades persistentes: ilusiones y desilusiones en el desarrollo humano y la integración social al quinto año del Bicentenario (2010-2014) (pp. 127-161). Buenos Aires, Argentina: EDUCA.

Salvia, A. (Coord.), Bonfiglio J. I., Donza E., Rodríguez Espínola S., Santángelo M. C. \& Vera, J. (2015). Progresos sociales, pobrezas estructurales y desigualdades persistentes: ilusiones y desilusiones en el desarrollo humano y la integración social al quinto año del Bicentenario (2010-2014). Buenos Aires, Argentina: EDUCA.

Scully, D., Kremer, J., Meade, M. M., Graham, R. \& Dudgeon, K. (1998). Physical exercise and psychological well-being: A critical review. British Journal of Sports Medicine, 32(2), 111-120. https://doi.org/10.1136/bjsm.32.2.111

Serra Grima, R. \& Bagur Calafat, C. (2004). Prescripción de ejercicio físico para la salud. Barcelona, España: Paidotribo.

Stephens, T. (1988). Physical activity and mental health in the United States and Canada: Evidence from four population surveys. Preventive Medicine, 17, 35-47. https://doi.org/10.1016/0091-7435(88)90070-9

Strawbridge, W. J., Deleger, S., Roberts, R. E. \& Kaplan, G. A. (2002). Physical activity reduces the risk of subsequent depression for older adults. American Journal of Epidemiology, 156, 328-334. https://doi.org/10.1093/aje/kwf047

Ströhle, A. (2009). Physical activity, exercise, depression and anxiety disorders. Journal of Neural Transmission, 116, 777-784. https://doi.org/10.1007/s00702-008-0092-x

Szretter Noste, M. E. (2017). Apunte de regresión lineal. Buenos Aires, Argentina: Universidad de Buenos Aires. Extraído de http://mate.dm.uba.ar/ meszre/apunte_regresion_lineal_szretter.pdf

Torres Deik, M., Moyano-Díaz, E. \& Páez, D. (2014). Comportamiento juvenil universitario en busca de la felicidad: su caracterización y su eficacia. Universitas Psychologica, 13, 1419-1428. https://doi.org/10.11144/Javeriana.UPSY13-4.cjub

Vagetti, G. C., Barbosa Filho, V. C., Moreira, N. B., de Oliveira, V., Mazzardo, O. \& de Campos, W. (2014). Association between physical activity and quality of life in the elderly: A systematic review, 2000-2012. Revista Brasileira de Psiquiatria, 36, 76-88. https://doi.org/10.1590/1516-4446-2012-0895

World Health Organization (2012). Physical activity. Genève, Suiza: Autor. Extraído de https:/www.who.int/mental_health/mhgap/evidence/depression/q6/en/

World Health Organization (2017). Depression and other common mental disorders: Global health estimates. Genève, Suiza: Autor.

Fecha de recepción: Mayo de 2018.

Fecha de aceptación: Febrero de 2019. 